\title{
Evolution of Size and Shape in the Colloidal
}

Crystallization of Gold Nanoparticles

Owen C. Compton and Frank E. Osterloh

Supporting Information (7 pages) 


$$
\begin{array}{ll}
\text { Equation 1: } & {\left[A_{1}\right]=\left[A_{1}\right]_{0} \exp \left(-k_{1} t\right)} \\
\text { Equation 2: } & {\left[A_{2}\right]=\frac{k_{1}\left[A_{1}\right]_{0}}{k_{2}-k_{1}}\left[\exp \left(-k_{1} t\right)-\exp \left(-k_{2} t\right)\right]} \\
\text { Equation 3: } & {\left[A_{4}\right]=\frac{k_{2}\left[A_{2}\right]_{0}}{k_{3}-k_{2}}\left[\exp \left(-k_{2} t\right)-\exp \left(-k_{3} t\right)\right]} \\
\text { Equation 4: } & {\left[A_{8}\right]=\frac{\left[A_{4}\right]_{0}}{k_{3}-k_{2}}\left[k_{3}\left(1-\exp \left(-k_{2} t\right)\right)-k_{2}\left(1-\exp \left(-k_{3} t\right)\right)\right]}
\end{array}
$$

These equations are derived from the differential rate equations for each species. The results are similar to that of the steady state approximation, but provide values for the concentrations of the intermediates $\mathrm{A} 2$ and A4. The results of this model, terminated at the octamer, are shown in Figure $2 \mathrm{C}$ in the manuscript.

Equation 1 (concentration of single particles): $[\mathrm{A} 1]_{0}$ is given by the initial number of gold nanoparticles in the colloidal gold solution $\left(2.14 \times 10^{14}\right)$. The rate constant $\mathrm{k} 1=0.1 \mathrm{~s}-1$ is obtained from a fit of [A1] to the DLS data.

Equation 2 (concentration of particle dimers): The first term accounts for the formation of dimers from single particles (k1) and the second term considers the loss of dimers due to formation of tetramers (k2).

Equation 3 (concentration of particle tetramers): see equation 2, with $\mathrm{k} 2$ for the formation and $\mathrm{k} 3$ for the disappearance of tetramers. [A2] $]_{0}$ was assumed to be $1.96 \times 10^{13}$ (the number of dimers after one second of reaction as predicted by Equation 2).

Equation 4 (concentration of octamers): In this equation, $[A 4]_{0}$ was assumed to be $1.49 \times 10^{12}$ (the number of tetramers formed after one second of reaction as predicted by Equation 3). 
Figure S-1




Figure S-1 (continued)

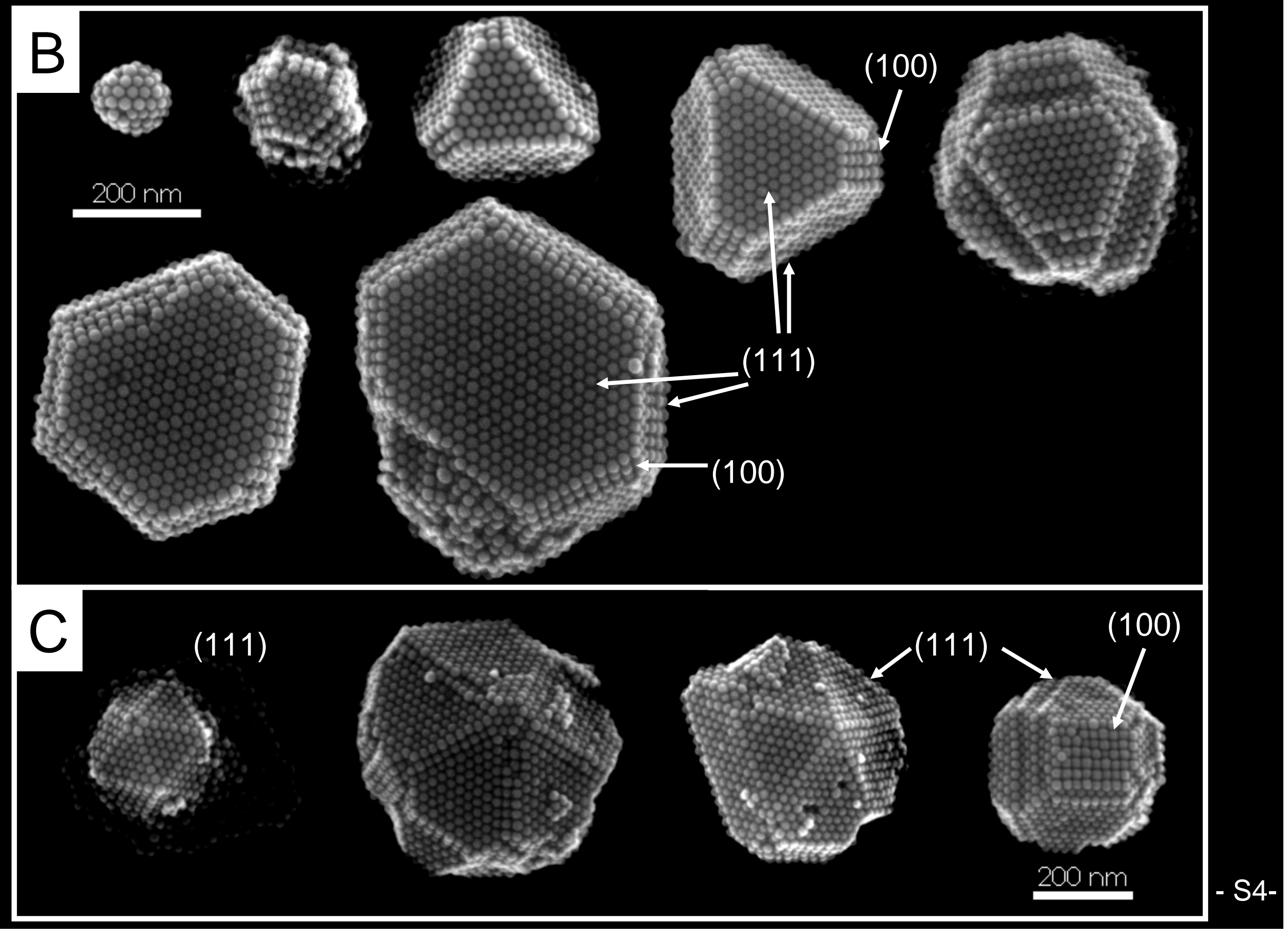


Figure S-1 (continued)




Figure S-2

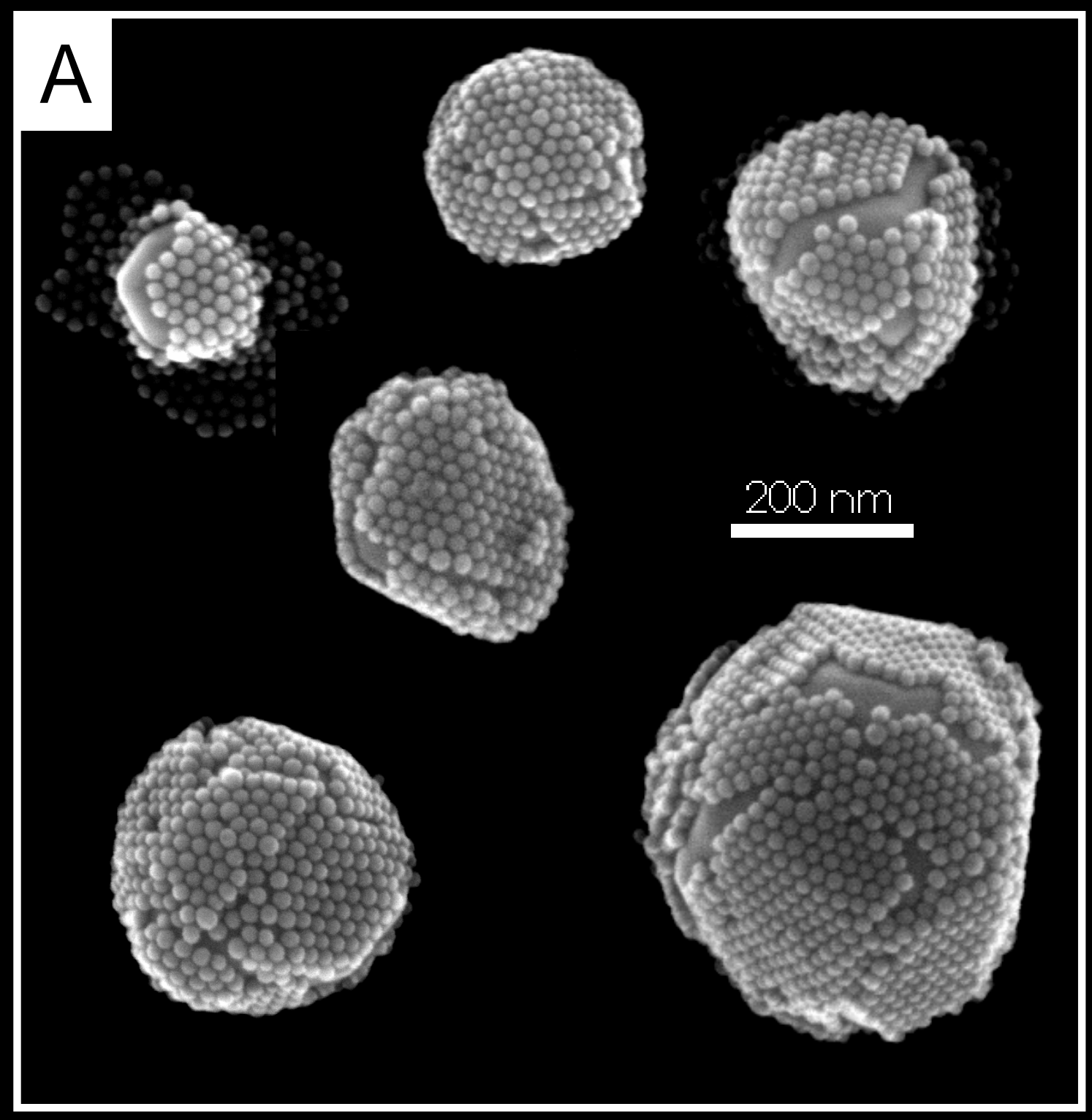


Figure S-2 (continued)

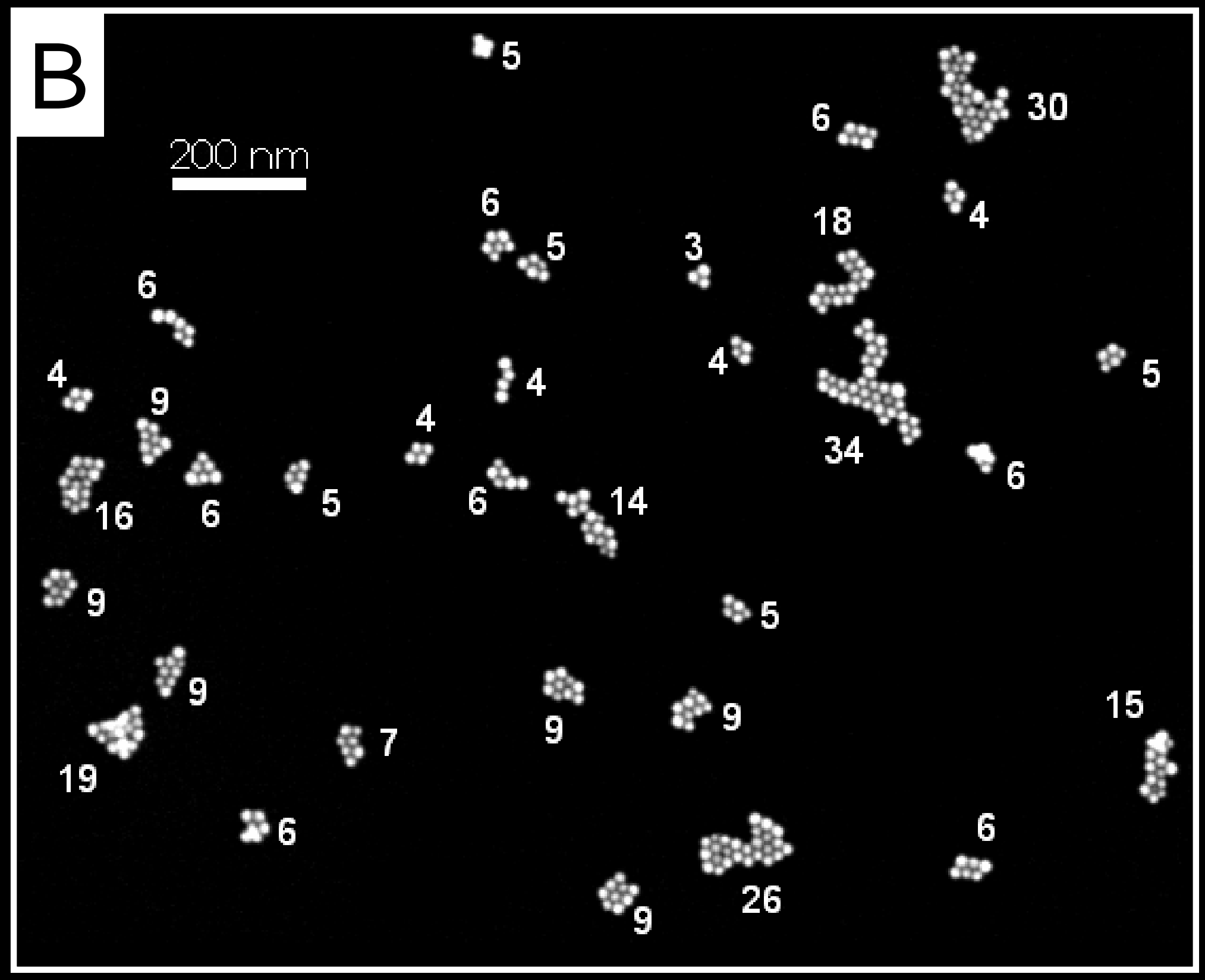

